\title{
Biochemical sensor based on a novel all-fibre cavity ring down spectroscopy technique incorporating a tilted fibre Bragg grating
}

\author{
Kaiming Zhou ${ }^{1}$, David Webb ${ }^{1}$, Mark Farries ${ }^{2}$, Neil Hayes ${ }^{3}$, Lin Zhang ${ }^{1}$, Ian Bennion ${ }^{1}$ \\ 1 Photonic Research Group, Aston University, B4 7ET, United Kingdom k.zhou@aston.ac.uk \\ 2 Gooch \& Housego Torquay Ltd, TQ2 7QL, United Kingdom \\ 3 EvanesCo Ltd, Newton Abbot, TQ12 4AD United Kingdom
}

\begin{abstract}
A novel all-fibre cavity ring down spectroscopy technique is proposed where a tilt fiber Bragg grating (TFBG) or long period grating (LPG) in the cavity provides sensitivity to surrounding medium. Such configuration with a LPG as the representative was theoretically analyzed. Two spectral bands were identified employable for sensing of surrounding refractive index for a weak LPG while only one band existed for a strong LPG. A TFBG, with enhanced sensitivity compared to usual LPGs, was used in a ring down cavity of $1 \mathrm{~m}$ constructed with 2 fiber Bragg gratings as the reflectors and the decay time changed from $220 \mathrm{~ns}$ to $450 \mathrm{~ns}$ when the TFBG was immersed into water from air.
\end{abstract}

keywords: Cavity ring down, long period grating, tilted fiber Bragg grating, optical fiber sensor

\section{Introduction}

Cavity ring down was first developed to evaluate mirrors of high reflectivity which are otherwise difficult to characterize [1]. Nevertheless, it was later found to be a suitable method to detect very weak losses in a cavity which could be caused by absorption of molecules of trace gases [2], and since then cavity ring down spectroscopy (CRDS) has become an important tool to analyze gas-phase materials[3]. Many aspects of CRDS have been developed, such as the working wavelengths, the useable targets and the cavity configuration, etc. As far as the latter point is concerned, the literature covers cavities formed by mirrors[2], prism[4,5], and also in optical fibres[6-11]. Recently, CRDS schemes constructed from optical fibres have become more and more of interest because alignment difficulties are removed and long interaction lengths are possible. Optical fibre based CRDS systems were initially constructed by placing mirrors formed by dielectric films at the two ends of the fibre but the desirability of connecting the cavity to other fibre can bring unwanted effects to these films, such as pressure-induced refractive index change of the film [11]. With the emergence of fibre Bragg gratings (FBGs), dielectric films could then be replaced with a pair of FBGs that act as wavelength selective mirrors made inside the fibre [9]. A fibre loop based ring down scheme to measure pressure has also been reported recently [7].

However, so far applications of fibre-based CRDS have been limited because the light in the core of the fibre is well screened from the target material surrounding the fibre. Some solutions to this problem have been brought forwards. Stewart and coworkers used a micro-optical cell in the fibre link[8], but by doing so the advantages of an all fibre device were compromised and active fibre had to be used to compensate for the extra loss incurred. Etching or tapering the fibre in the cavity to expose the evanescent wave were also reported, but the configuration then becomes fragile [11]. In this paper, we report on a novel method in which sensitivity to surrounding refractive index is provided by incorporating a long period grating (LPG) or tilted fibre Bragg grating (TFBG) within the cavity.

\section{Theoretical analysis}

A LPG is a fibre grating structure that couples light of certain wavelengths from the core mode into forwardpropagating cladding modes from where the light is lost by scattering and absorption. LPGs reveal themselves as a series of attenuation bands in the transmission spectrum of the core guided light. Although backward-propagating cladding modes could also be excited by FBGs, we will concentrate on the forward coupling regime in this paper and leave the backward coupling to future work. Besides LPGs and FBGs, another less familiar grating type, the TFBG, is also capable of coupling light from the core mode into both forward and backward propagating cladding modes, depending on the angle of tilt; these structures possess some unique properties that make them attractive for some sensing tasks[12]. When the tilt angle of the grating is big enough, mode coupling to forward-propagating cladding modes is enabled, and thus the device can be considered to be a type of LPG.

Coupled mode theory (CMT) is usually employed to analyze and simulate the optical properties of fibre gratings. For TFBGs, a Green's function (or volume-current method) is also employed but is only useful for radiation mode 
coupling [13]. We use commercially available software, IFO from Optiwave which is based on CMT, to simulate the gratings used in our scheme.

Figure 1 depicts our ring down configuration incorporating a LPG/TFBG in a cavity formed with two FBGs as the mirrors. The LPG/TFBG couples the light from the core to the cladding and hence gives rise to loss from the core mode which is dependent on the properties of the surrounding medium, such as the refractive index and absorption. When these properties change, the loss varies and consequently causes a change in the ring-down time. In this work, we use the refractive index of the surrounding medium as an example to demonstrate this approach. To be compatible with the CRD system, the loss induced by the LPG/TFBG should be very small otherwise the ringdown time would be too short to measure. There are several ways to achieve this aim. We could use either very weak gratings or the far edges of the gratings where the loss is inherently low. Alternatively, for sufficiently strong LPGs, the loss at the central portion of the spectrum could also become low enough when the strength is such that the light is coupled back to the core by the rear part of the gratings. Here we will study the losses induced by a weak grating and the relation of the ring down time to the surrounding refractive index (SRI). [11]:

The parameters of the LPG structure used in the simulation are listed in table 1. The ring down time is given by

$$
\tau=\frac{l \cdot n_{e f f}}{c \cdot(1-T+1-R)}
$$

where the meanings of the symbols and their values in the simulation are also given in table 1 . Figure $2 \mathrm{a}$ gives the transmission spectra of the LPG for different SRIs where we are taking coupling to the $\mathrm{LP}_{04}$ cladding mode as an example. As can be seen from the figure, when the refractive index changes, the profile of the attenuation band of the LPG shifts and at a given wavelength the loss changes accordingly. Figure $2 b$ shows calculated ring down time as a function of wavelength obtained using equation 1 for each SRI. Though ring down time variation is also noticeable for the side bands, the main band gives the most significant response. There exist two regions either side of the main peak, band 1 and band 2, within which each wavelength could be employed to test SRI since only in these two regions does the loss monotonically decrease or increase with respect to significant changes in SRI. Band 1 is from wavelength A, which corresponds to the left zero of the main peak for the lowest SRI, to wavelength B which is the minimal point of the main peak for the highest SRI curve. Similarly, wavelength C and D of band 2 can also be determined. The bandwidth of band 1 and 2 is approaching $50 \mathrm{~nm}$ and is much bigger than that of most FBGs. Figure $3 \mathrm{a}$ and $3 \mathrm{~b}$ give the ring down time versus the SRI for different wavelengths in these two bands. Obviously, the ring down time varies nonlinearly with SRI. Especially, when the SRI draws near to the index of the fibre, the ring down time varies dramatically and thus gives significant sensitivity which can be defined as variation of ring down time per refractive index unit (RIU). The largest sensitivity obtained is $150 \mu \mathrm{s} / \mathrm{RIU}$. If the ring down time resolution is $1 \mathrm{~ns}$, then the smallest index change detectable would be $6.5 \times 10^{-6}$. For band 1 , the ring down time decreases when the SRI is enlarged while for band 2, it increases.

Though high sensitivity could be attained at the high SRI region, the change in ring-down time at the left side of figure $3 \mathrm{a}$ and $3 \mathrm{~b}$ is relative small, especially for $3 \mathrm{~b}$. Most biomaterial analysis happens in water based solutions with refractive index around 1.33. In order to enable our scheme to work effectively for this kind of application, we need to optimize the grating. Simply increasing the grating's length and attenuating the strength accordingly to maintain the low loss, higher sensitivity in the low SRI region could in principle be achieved. In figure 4 is shown the ring down time versus SRI for a grating with a length of $5 \mathrm{~cm}$ and strength of $1.3 \times 10^{-5}$. Apparently, the scale of offset to the ring down time in the region from 1.3 to 1.42 is enlarged for both band a and $\mathrm{b}$. In figure $4 \mathrm{~b}$, some curves present an almost linear relation. However, for longer gratings, the employable bandwidth is narrowed to 4 or $5 \mathrm{~nm}$. But even so it is still bigger than that of FBGs.

\section{Experiments and Discussion}

A TFBG with a large tilt angle could be regarded as a kind of LPG with the benefits of low thermal cross sensitivity and insensitivity to bending [12]. Most importantly, they have significant sensitivity to SRI around that of water, i.e., 1.33. Figure $5 \mathrm{a}$ is the spectrum of one peak of the large angle TFBG at $1560 \mathrm{~nm}$ and Fig. $5 \mathrm{~b}$ shows the wavelength shift with respect to the SRI. The inset of fig. 5a is the fringes of the grating. As the figure shows, the sensitivity can reach up to $\sim 340 \mathrm{~nm} / \mathrm{RIU}$ around 1.33 . Thus we used a TFBG with tilt angle of $81^{\circ}$ in the cavity formed by a pair of FBGs with wavelength of $1543 \mathrm{~nm}$ and maximal reflectivity of $32 \mathrm{~dB}$ and $33 \mathrm{~dB}$. Figure 6 is the setup of the cavity ring down measurement. In the experiment, light comes from a tuneable laser and is then amplified by an EDFA. It is modulated by an AOM, which is controlled by a pulse generator, giving pulses of $250 \mathrm{~ns}$ and period of $2 \mu \mathrm{s}$, before being launched into the cavity. With a FBG filter, the sidebands of the source light which extend outside the 
reflectivity profile of the gratings forming the cavity are eliminated. Upon reaching the detector, the signal is collected and read from a digital oscilloscope.

Initially, the cavity length is about $1 \mathrm{~m}$-long with no TFBG included. Figure 7 a gives the tested decay curve and the ring down time is measured to be 550ns. The $\mathrm{x}$-axis in the figure is in sample points where 1 point corresponds to a time of $2 \mathrm{~ns}$. Theoretically, for such a cavity, the decay time should be above $1 \mu \mathrm{s}$ and this discrepancy could be explained by extra loss induced when UV laser inscription was performed.

The TFBG has to be spliced into the cavity and the influence of splicing needs to be characterized first. We broke the cavity from the middle and then spliced it, causing the ring down time to reduce to 220ns even though the splicer indicated 0dB loss (this suggests that CRDS could be usefully employed to characterise low loss splices). Thus the influence from splicing in the cavity is not trivial and needs to be compensated for. To this end, an extra $10 \mathrm{~m}$ fibre was spliced with the TFBG in the cavity and 3 splicing points were therefore introduced. When the TFBG is in air, the loss is huge and no decay curve could be observed because the spectral feature of the TFBG is just at the working wavelength shown by the vertical line in figure 5a. We then put the TFBG in water and its transmission spectrum is shown by the solid line in figure 5a: clearly the peaks shift to the right and the loss at $1543 \mathrm{~nm}$ is reduced to a large degree. We then measured the CRD signal and figure $7 \mathrm{~b}$ gives the CRD decay curve from which we determined a decay time of 450ns. When water is replaced with methanol, a slight increase to the average decay time was noticed. If materials with SRI from 1 to 1.33 were used, decay times from $0 \sim 450$ ns should be detectable. Because of the loss induced by the 3 splicing points, the sensitivity of the device is reduced significantly. In future work, gratings will be fabricated in a single piece of fibre and better results should be obtained.

\section{Conclusion}

With the aid of an evanescent wave induced by a TFBG, a novel fibre cavity ring down spectroscopy technique was demonstrated. Theoretical simulations have been carried out and encouraging results were achieved, predicting sensitivity as high as $6.5 \times 10^{-6} \mathrm{RUI} / \mathrm{ns}$. A novel biochemical sensor employing a TFBG with tilt angle of $81^{\circ}$ in the CRD cavity was demonstrated as a proof of concept and a ring down time of 450ns was attained when the sensing grating was in water.

5.Acknowledgement This work was carried out as part of the UK DTI funded Sentinel project and we acknowledge our project partners SIFAM Fibre Optics and EvanesCo for their technical support and useful discussions.

\section{References}

[1] J. M. Herbelin, J. A. McKay, M. A. Kwok, R. H. Ueunten, D. S. Urevig, D. J. Spencer, and D. J. Benard, Sensitive measurement of photon lifetime and true reflectances in an optical cavity by a phase-shift method, Appl. Opt. 1980;19:144-7

[2] O'Keefe and D. A. G. Deacon, “Cavity ringdown optical spectrometer for absorption measurements using pulsed laser sources,” Rev. Sci. Instrum. 1988;59:2544-51.

[3] Patent numbers: WO2005088274, WO2005088277, WO2004068123

[4] C. R. Pipino, J. W. Hudgens, and R. E. Huie, Evanescent wave cavity ring-down spectroscopy for probing surface processes, Chem. Phys. Lett. 1997; 280:104-12

[5] C. R. Pipino, J. W. Hudgens, and R. E. Huie, Evanescent wave cavity ring-down spectroscopy with a totalinternal-reflection minicavity, Rev. Sci. Instrum. 1997; 68: 2978-89 (1997).

[6] C.Wang and S. T. Scherrer, Fiber Loop Ringdown for Physical Sensor Development: Pressure Sensor, Appl. Opt. 2004;43:6458-64

[7] C.Wang and S. T. Scherrer, Fiber ringdown pressure sensors, Opt. Lett. 2004;29:352-4

[8] G. Stewart, K. Atherton, and B. Culshaw, Cavity-enhanced spectroscopy in fiber cavities, Opt. Lett. 2004;29: 442-4

[9] M. Gupta, H. Jiao, and A. O'Keefe, Cavity-enhanced spectroscopy in optical fibers, Opt. Lett. 2002;27:1878-80

[10] P. B. Tarsa, D. M. Brzozowski, P. Rabinowitz, and K. K. Lehmann, Cavity ringdown strain gauge, Opt. Lett. 2004;29: 1339-41

[11] T. V. Lerber and M.W. Sigrist, Cavity Ring-down Principle for Fiber optic Resonators: Experimental Realization of Bending Loss and Evanescent-field Sensing, Appl. Opt. 2002; 41:3567-75

[12] K. Zhou, L. Zhang, X. Chen, and I. Bennion, Optic sensors of high refractive-index responsivity and low thermal cross sensitivity that use fiber Bragg gratings of $>80^{\circ}$ tilted structures, Opt. Lett. 2006;31:1193-95

[13] Y. Li, M. Froggatt, and T. Erdogan, Volume Current Method for Analysis of Tilted Fiber Gratings, J. Lightwave Technol. 2001,19:1580-91 
Figure Captions:

Figure 1 CRDS configuration incorporating a LPG/TFBG in cavity

Figure 2 (a) The transmission spectra of the LPG for different SRI. (b) calculated ringdown time from (a).

Figure 3 Ring down time versus the SRI for different wavelengths in band (a) 1 and (b) 2

Figure 4 ring down time versus the SRI for different wavelengths in band (a) a and (b) b for a long grating

Figure 5 (a) transmission spectrum of one peak of an $81^{\circ}$ TFBG. Dotted line is for SRI=1.0 and solid line is for SRI of 1.33. Vertical line shows wavelength of operation. Inset is the fringes of this grating (b) Wavelength shift of the peak versus the refractive index surrounding the grating.

Figure 6 Setup of cavity ring down Experiment

Figure 7 (a) ring down curve of a pristine $1 \mathrm{~m}$ cavity with ring down time of 550ns (b) ring down curve of a $11 \mathrm{~m}$ cavity with an $81^{\circ}$ TFBG in water, which gives a ring down time of $450 \mathrm{~ns}$

Table 1

\begin{tabular}{|l|c|c|c|c|c|c|c|c|c|c|}
\hline Symbol & $L$ & $\Lambda$ & $\delta n$ & $a$ & $b$ & $l$ & $n_{\text {eff }}$ & $c$ & $T$ & $R$ \\
\hline Meaning & $\begin{array}{c}\text { LPG's } \\
\text { Length }\end{array}$ & $\begin{array}{l}\text { LPG's } \\
\text { Period }\end{array}$ & $\begin{array}{c}\text { refractive } \\
\text { index change }\end{array}$ & $\begin{array}{c}\text { fiber } \\
\text { core's } \\
\text { Diameter }\end{array}$ & $\begin{array}{c}\text { fiber's } \\
\text { Diameter }\end{array}$ & $\begin{array}{c}\text { Cavity } \\
\text { length }\end{array}$ & $\begin{array}{c}\text { Effective } \\
\text { index }\end{array}$ & $\begin{array}{c}\text { Light } \\
\text { velocity }\end{array}$ & $\begin{array}{c}\text { Cavity's } \\
\text { Transmission }\end{array}$ & $\begin{array}{c}\text { Effective } \\
\text { reflectivity of } \\
\text { the mirrors }\end{array}$ \\
\hline Value & $5 \mathrm{~mm}$ & $450 \mu \mathrm{m}$ & $1 \times 10^{-4}$ & $8.3 \mu \mathrm{m}$ & $125 \mu \mathrm{m}$ & $1 \mathrm{~m}$ & 1.44628 & $3 \times 10^{8} \mathrm{~m} / \mathrm{s}$ & Variable & 0.999 \\
\hline
\end{tabular}




\begin{tabular}{ccc} 
FBG1 & LPG/TFBG & FBG2 \\
\hline \hline
\end{tabular}

revised figure 1
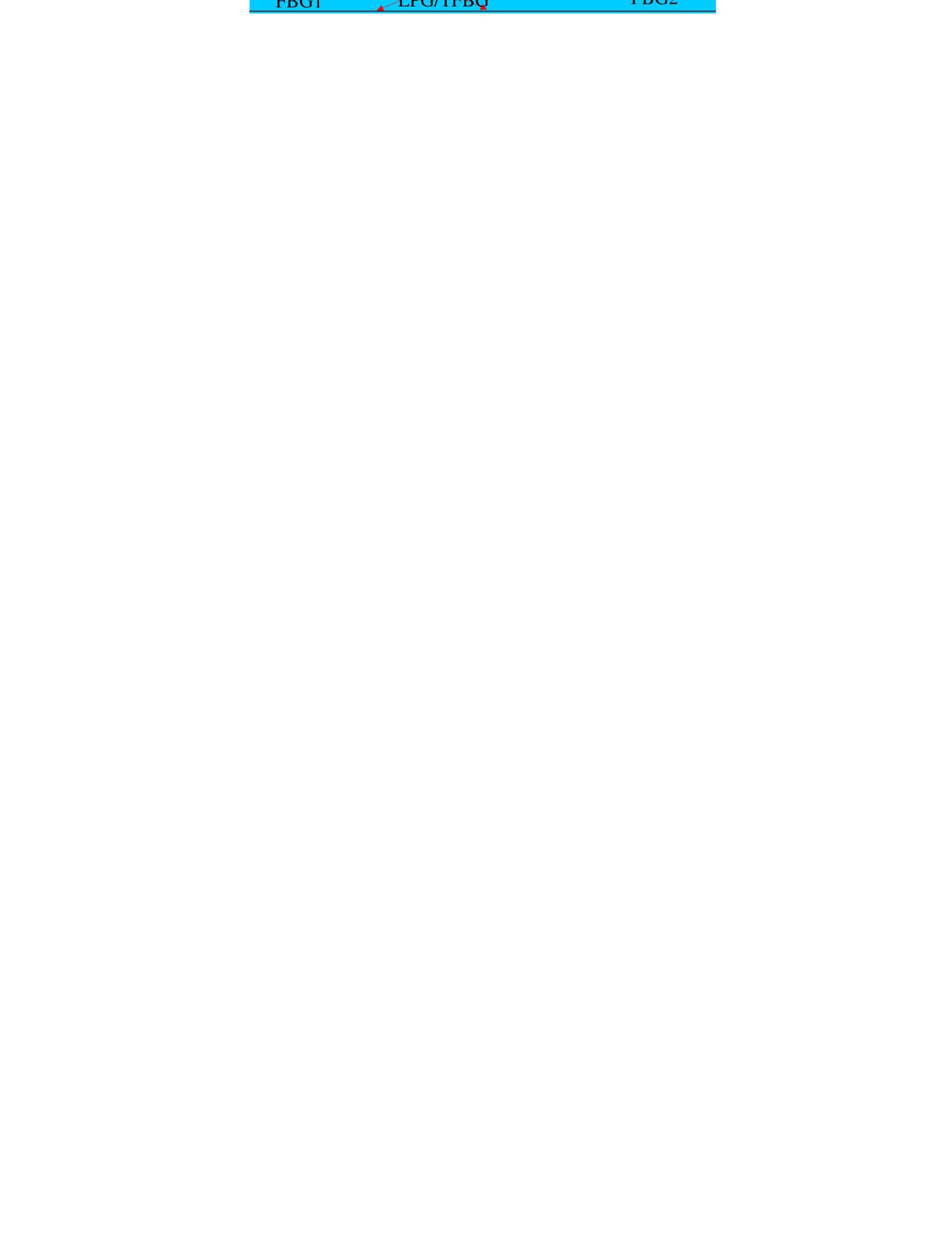


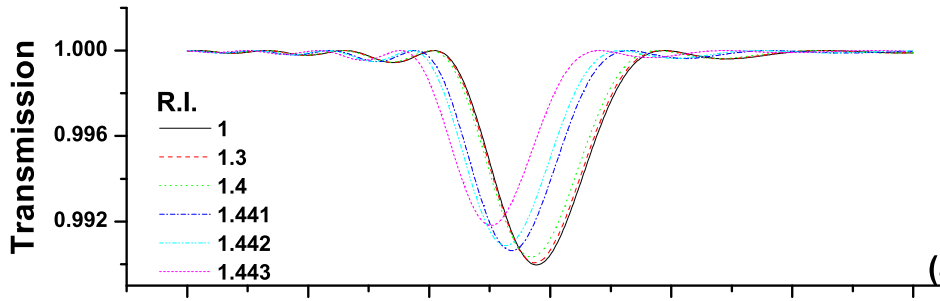

(a)



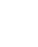




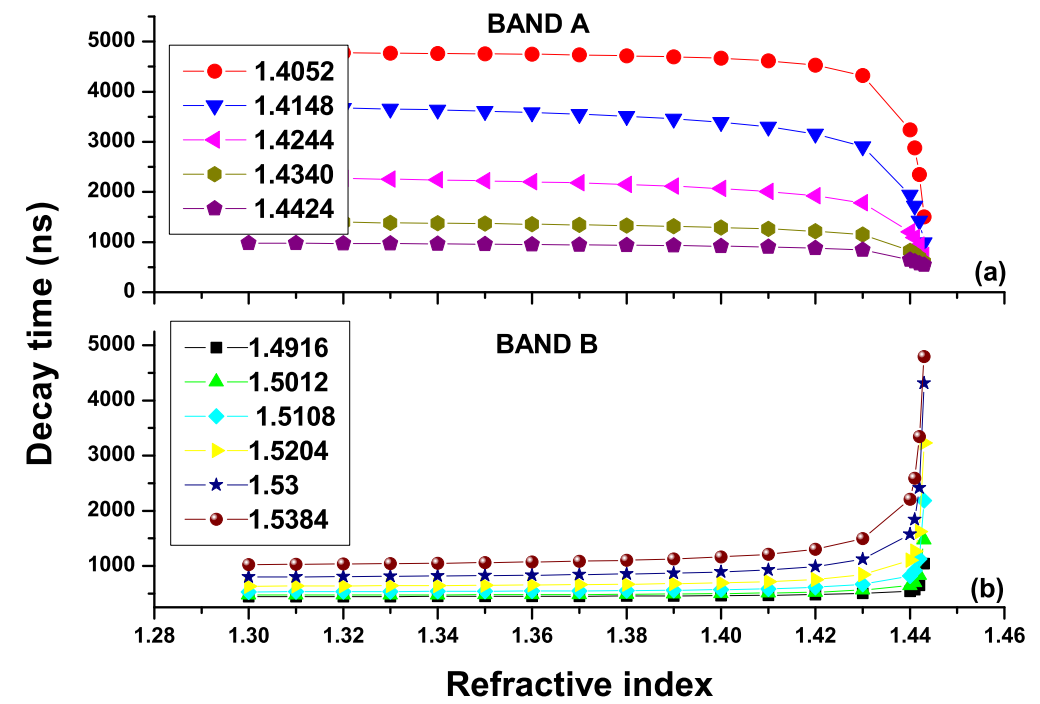

Refractive index 


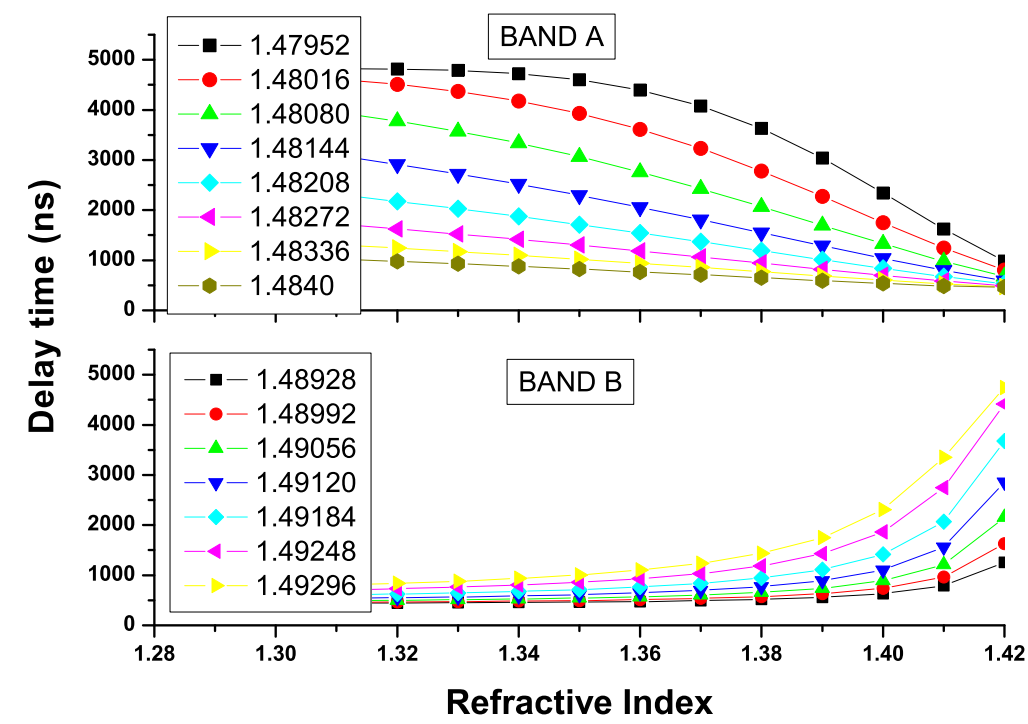




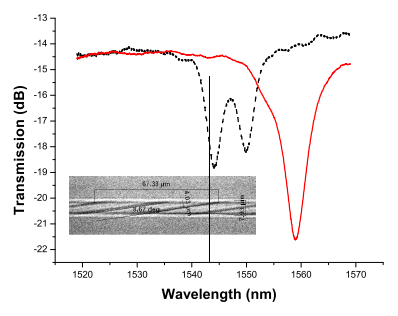

(a)

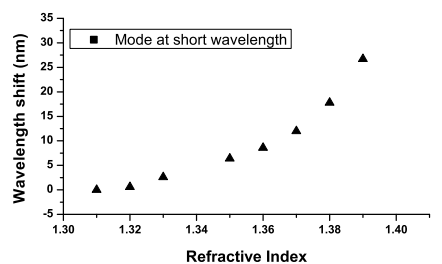

(b) 


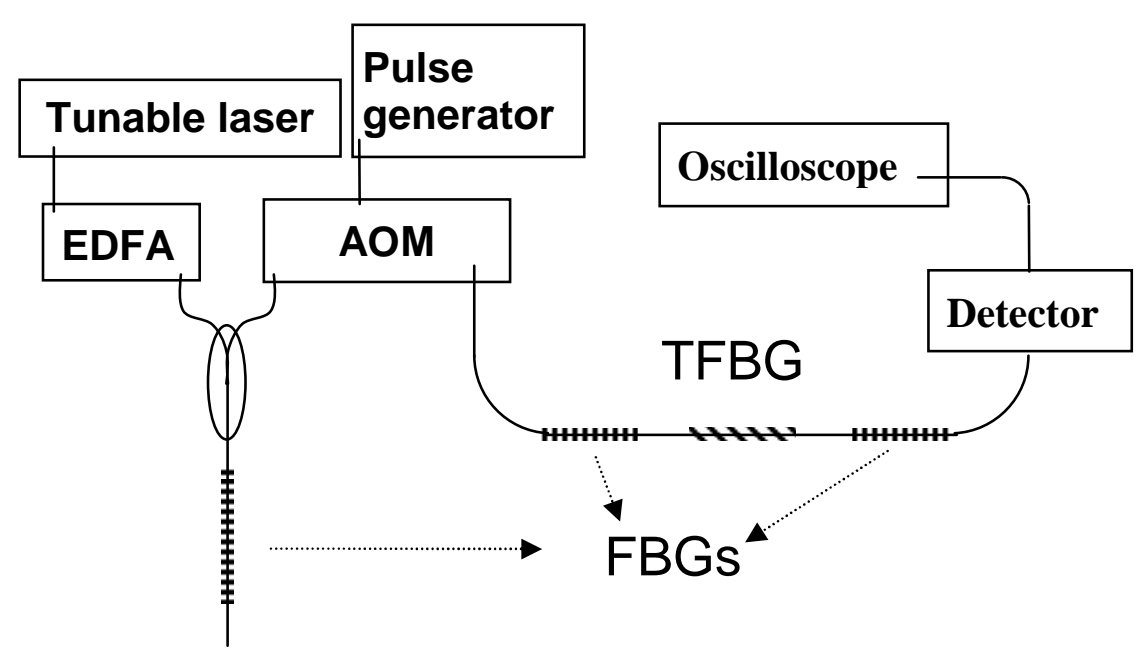

Pulse
Oscilloscope

TFBG

Detector 




(a)

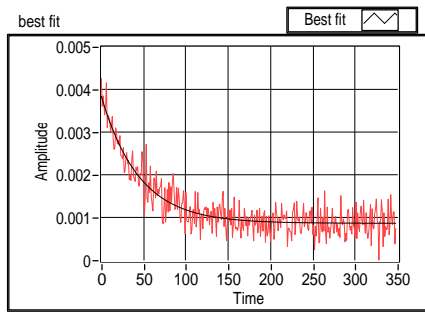

(b) 